\title{
Colecciones de narrativa folklórica argentina y eslovena: una comparación
}

\author{
María Inés Palleiro \\ Universidad de Buenos Aires/ Consejo Nacional de Investigaciones \\ Científicas y Técnicas, Argentina \\ marinespalleiro@gmail.com
}

Recibido: 7/3/2020. Aceptado: 7/7/2020.

\begin{abstract}
Resumen
La Argentina y Eslovenia son jóvenes naciones cuyos archivos de narrativa folklórica tienen aspectos en común. En este artículo, se analizan algunos de estos aspectos, a partir de un recorrido diacrónico por colecciones de relatos de ambos países, cuyas modalidades de ordenamiento reflejan la incidencia de distintos paradigmas del Folklore, entendido como expresión estética de identidades sociales. El recorrido intenta destacar el valor de los archivos del Folklore para configurar memorias sociales, no solo mediante la preservación patrimonial del pasado, sino también por su actualización en el presente. La tradición custodiada en archivos es concebida como fuerza transformadora que resignifica identidades y sirve como instrumento de cambio social. Su sistematización y difusión, acompañada de una reflexión epistemológica sobre los paradigmas que los sustentan, estimula el diálogo entre culturas, indispensable para situar expresiones identitarias nacionales, como la argentina y la eslovena en un mapa global.
\end{abstract}

\section{Argentinean and Slovenian folk narrative collections: a compara- tive approach}

\begin{abstract}
Argentina and Slovenia are young nations whose folk narrative archives share distinctive features. This article analyzes some of these distinctive features, departing from a diachronic overview of folk narrative collections of both countries. The way of organizing collections in both cases shows the influence of different trends in Folklore studies, understanding Folklore as an aesthetic expression of social local identities. The analytic approach to Argentinean and Slovenian collections is aimed to highlight the relevance of the folk narrative archives to construct traditions and social memories, not only by preserving the heritage of the past but also by updating traditions in the present. From this point of view, tradition, as kept in archives, is conceived as a transformative force that resignifies identities and serves as an instrument of social
\end{abstract}

Palabras clave

narrativa folklórica La Argentina

Eslovenia archivos identidades sociales

Keywords

folk narrative Argentina Slovenia archives social identities 
1. Este centro de investigación, el más antiguo de Eslovenia, data de 1693, cuando la Academia Operosorum de los jesuitas se estableció en Ljubljana (Koiva y Kupernajov, 2004). changes. Thus, the comparative studies of folk narrative collections of different countries, along with the consideration of the epistemological paradigms which sustain them, stimulates the dialogue between cultures, indispensable for locating national identity expressions, such as Argentinian and Slovenian ones, in a global map.

\section{A modo de introducción}

Este artículo tiene por objeto sentar las bases para una comparación diacrónica de archivos y colecciones de narrativa tradicional argentina y eslovena. Tal comparación se encuadra en un proyecto más amplio, del que participan, por una parte, las investigadoras eslovenas Barbara Ivančič Kutin y Monika Kropej Telban y, por otra, el equipo de trabajo coordinado por Aída González, de la Universidad Nacional de San Juan, directora del Instituto de Investigaciones Lingüísticas y Filológicas "Dr. Manuel Alvar", en el que se domicilia el "Fondo Berta Elena Vidal de Battini" (en adelante, FONVIBA). En el marco de este proyecto, coordinado por quien escribe, las investigadoras eslovenas se dedicaron a profundizar el estudio de archivos y colecciones de ese país (Ivančič Kutin en Palleiro comp. in press y Kropej Telban en Palleiro comp. in press), y el equipo de Aída González tuvo a su cargo la descripción y comentario de la totalidad del material que integra el FONVIBA, al que se hará referencia más abajo.

La presente contribución está focalizada entonces, de modo predominante, en el estudio diacrónico de archivos y colecciones de la Argentina, con especial énfasis en la colección de Berta Elena Vidal de Battini - por tratarse del corpus que reúne la mayor cantidad de manifestaciones de narrativa folklórica de todo el país-, como punto de partida para el trazado de lineamientos comparativos que sirvan como base para su integración con los frutos del trabajo de las citadas investigadoras

\section{Colecciones de narrativa folklórica argentina y eslovena: una com- paración}

La Argentina y Eslovenia son jóvenes naciones cuyos archivos de narrativa folklórica tienen aspectos comunes, advertidos durante una estadía sabática en el Institute of Slovenian Ethnology de la Slovenian Academy of Sciences and Arts (SASA), ${ }^{1}$ financiada por el Consejo Nacional de Investigaciones Científicas y Técnicas en 2017-2018.

\section{1. ¿Cómo surgió este intercambio?}

El primer contacto con estudiosas eslovenas surgió en Atenas en 2009, en una reunión científica internacional dedicada al estudio de la narrativa folklórica. Allí quien esto escribe tomó conocimiento de las investigaciones de Barbara Ivančič Kutin y Monika Kropej Telban, y así nació el proyecto de comparar archivos folklóricos argentinos y eslovenos, cuyos lineamientos iniciales fueron sistematizados en una publicación (Palleiro y Civila Orellana, 2015). La experiencia continuó con un trabajo de campo realizado con Ivančič Kutin en enero 2015 en la frontera ítalo-eslovena del valle del río Soča y región alpina de Bovec y Tolmin. Se partió de Gorizia, Italia, para cruzar luego la frontera de Nova Gorica hasta Bovec, donde registramos celebraciones de carnaval (Palleiro, 2017). Este itinerario dio lugar a la visita a museos y talleres de artesanos, en los que pudieron advertirse similitudes con la producción del artesano riojano Marino Córdoba, por sus tallas y estatuillas cerámicas de duendes y otros seres sobrenaturales. ${ }^{2}$ Se registraron relatos, canciones y otras expresiones tradicionales en italiano y esloveno. El viaje finalizó con una visita al museo de Kobarid, con su sala de banderas de Eslovenia, que pasó del dominio austro-húngaro al italiano y 
yugoslavo, hasta su surgimiento como nación independiente, con una mixtura cultural que es un aspecto común con la cultura argentina. En este viaje de campo, se hicieron evidentes las semejanzas entre el paisaje cultural de Alpes y Andes patagónicos que, como lo confirmó la visita a la Argentina de Ivančič Kutin, fue factor decisivo para el asentamiento de inmigración eslovena en zonas como Bariloche.

En 2016, tuvo lugar la primera visita de quien esto escribe a los archivos de narrativa folklórica del Slovenian Ethnology Institute (SASA) de Ljubljana. Tal visita fue una oportunidad para tomar contacto con la clasificación tipológica de relatos desarrollada por Kropej Telban, y madurar a partir de tal contacto la posibilidad de una tarea similar con relatos argentinos. La segunda visita, realizada en enero 2017, permitió delinear un proyecto sabático. Con la colaboración de Ivančič Kutin y Kropej Telban, fueron consultados tanto el archivo de narrativa tradicional eslovena de Dolenc, como el material señero de Maticetov de los años '50, y la magna colección édita de narrativa folklórica eslovena (1988-2020) de Stanonik, a quien se logró entrevistar. Esta fue también una ocasión para trabajar con antologías de relatos folklóricos eslovenos de Kropej Telban et al. (2010), que brindaron elementos para la tarea comparativa.

\section{La nación eslovena}

El actual territorio esloveno fue habitado en tiempos prerromanos, según Šavli (2004), por pobladores de Iliria y regiones célticas y en el primer siglo AC integró el Imperio romano. Un grupo eslavo fundó Samu en VI DC y dominó Hungría hasta que Carlomagno lo subordinó en el siglo VIII. Luego de la derrota de los húngaros por los turcos en 1526, aceptó la dominación austríaca para evitar la turca. Al igual que Croacia, es un país católico romano y estas creencias, reflejadas en relatos folklóricos, constituyen un punto de contacto con la narrativa tradicional argentina. Con el derrumbe del imperio austrohúngaro, Eslovenia comenzó a forjar su independencia. Formalmente se unió con Montenegro, Serbia, y Croacia en 1918, y se integró a Yugoslavia en 1929. Durante la Segunda Guerra Mundial, Alemania ocupó Yugoslavia y Eslovenia fue dividida entre Alemania, Italia, y Hungría. Después de 1945, fue convertida en república comunista yugoslava. En la década de1980, comenzó a recuperar autonomía y declaró su independencia en 1991, con escasa violencia. La Comunidad Europea reconoció tal independencia en 1992 y Eslovenia se unió a ella en 2004. Su historia de subordinaciones, con un impacto diferente en las diversas regiones, le confirió una identidad plural que guarda similitudes con el entramado intercultural de la Argentina, cuya identidad también se configuró a partir del mosaico de las diferencias regionales, de acuerdo con el principio de unidad en la diversidad. En el marco de este resurgimiento de Eslovenia como nación independiente, se renovó el interés por recuperar sus tradiciones folklóricas, en un proyecto político y cultural orientado a reconstruir aspectos de la identidad nacional, a partir de la integración de las identidades locales. ${ }^{3}$ Tal propósito de integración de particularidades regionales en torno a un concepto de nación, plasmado en un proyecto político y educativo que reconoce las diversidades locales fue también el propósito que guió la tarea de recopilación de material folklórico argentino, iniciado en el año 1921 por parte de instituciones como el Consejo Nacional de Educación, y continuado por estudiosos como Berta Elena Vidal de Battini, Inspectora General de Escuelas, a quien se hará referencia en una sección siguiente de este trabajo.

\section{Archivos de narrativa folklórica eslovena: recorrido diacrónico}

En su visita a la Argentina en 2019, Ivančič Kutin dio una conferencia en el Instituto de Filología y Literaturas Hispánicas «Amado Alonso» de la Universidad de Buenos Aires, y presentó un panorama diacrónico de colecciones y archivos eslovenos. Destacó que
3. Una veta fecunda para futuras investigaciones, que excede los límites de este artículo, es el análisis de las similitudes y diferencias de este proceso de construcción de la tradición nacional argentina y eslovena. Para un análisis del proceso de construcción de la tradición nacional y nativista en obras como la de Joaquín V. González, ver Chein (2011: 27-30 y 38). Para un agudo análisis del vínculo entre Folklore, nacionalismo, nativismo, criollismo e interculturalidad, ver Chamosa (2010, 2012). Para un abordaje del valor de las tradiciones locales para la forja de la identidad cultural eslovena, ver Ivančič Kutin (2010). 
4. Para una referencia a la obra de Baudouin de Courtenay, iniciador de la llamada "geografía folklórica”, ver Skubik (2015: 167-168.) Para una referencia al impacto de las investigaciones de Baudouin de Courtenay en los estudios folklóricos, ver Koiva y Kuperjanov (2005).

5. Para una referencia a la obra de Gašper Križnik, ver Stanonik et al.(2008).

6. Las características de este archivo organizado por Orel se encuentran descriptas en el sitio del Institute of Slovenian Ethnology (https:// isn2.zrc sazu.si/sl/zbirka/zbirkaslovenskih-folklornih-ugank\#v).

7. En su viaje a Argentina, Ivančič Kutin registró material folklórico de migrantes eslovenos para esa colección. el primer recolector de narrativa tradicional fue Jan Baudouin de Courtenay (1845-1929), quien encaró entre 1872-1873 una investigación sobre dialectos (Baudouin de Courtenay, 1910), y relevó material folklórico en Rezija, Oeste de Eslovenia. ${ }^{4}$ Invitó en periódicos a que sus lectores recopilaran material dialectológico y folklórico, de acuerdo con instrucciones, como ocurriría en la Argentina con la Encuesta folklórica de 1921. El material recopilado se encuentra en la Academia de Ciencias de San Petersburgo (Rusia). Por la misma época, Gašper Križnik (1875: 67-104) zapatero de Gorenjska (nacido en 1849 y muerto en 1904) registró por vez primera folklore esloveno en dialecto. Por su parte, Karel Štrekelj (1859-1912) se interesó en el cancionero popular, y registró 8686 canciones -con metadatos como fecha y lugar de recolección, nombre del recolector, y, en textos escritos, fuente édita o manuscrita-. Publicó estos registros en cuatro tomos entre 1895 y 1923, en una tarea similar a la emprendida en la Argentina por Juan Alfonso Carrizo (1926-1947). También Milko Matičetov (1819-1914) registró cuentos folklóricos en la zona del valle del río Soča que recorrimos con la Dra. Ivančič Kutin en nuestro viaje de campo. Más adelante, el profesor Dolenc (1926-2012) reunió entre sus alumnos de la escuela de Tolmin (localidad que visitamos con la Dra. Ivančič Kutin) material narrativo folklórico entre 1956 y 1990, del que se conserva también archivo sonoro. Dicho material contiene metadatos como lugar y momento de registro, y nombre de recolectores e informantes.

El Institute of Ethnology de la Slovenian Academy of Sciences and Arts domicilia también una colección electrónica de 2500 adivinanzas, 60000 proverbios y cine etnográfico, y el Museo Etnográfico Esloveno conserva material sobre costumbres, comidas, canciones y relatos tradicionales de 18 trabajos de campo organizados por Boris Orel, director del Museo Etnográfico de Eslovenia, entre 1948 y $1961 .{ }^{6}$ Este archivo, que consta de cuestionarios y cuadernillos de campo, guarda similitud con el del Museo Nacional de Antropología y Pensamiento Latinoamericano que domicilia la Encuesta folklórica de 1921, y con el del Instituto de Investigaciones Lingüísticas y Filológicas «Manuel Alvar» (en adelante, INILFI), Universidad Nacional de San Juan, que domicilia el Fondo «Berta Elena Vidal de Battini» (FONVIBA) y registros de campo de equipos de César Quiroga Salcedo y Aída González.

El archivo más extenso de narrativa folklórica eslovena es la colección Glasovi (Voices), dirigida por Marija Stanonik (nacida en 1947), de 51 volúmenes, publicados desde 1988 hasta hoy, con nuevas obras en preparación. Contiene 20.180 relatos de 4178 narradores, registrados por 746 recolectores. Cada libro cubre una zona diferenciada de Eslovenia y proyecta incluir relatos de migrantes eslovenos de Europa, América y Australia. ${ }^{7}$

De los archivos recientes, se destaca la publicación Krivopete de Ivančič Kutin (2018). Las Krivopete, definidas en testimonios orales como "mujeres salvajes con pies al revés" (Ivančič Kutin 2018: 171) tienen su correlato en el Folklore del Nordeste argentino y latinoamericano en la Cai-Pora. La investigadora, que describe las Krivopete como "mascotas", símbolos identitarios de regiones alpinas, incluye documentación procedente de archivos de narrativa tradicional eslovena de los siglos XIX y XX cuya cronología trazó en su conferencia, complementada con material de campo recopilado entre 2014 y 2017 en el valle del Soča y en Friuli-Venezia Giulia, Italia.

Los archivos del Instituto esloveno nutrieron también investigaciones de Kropej Telban, quien editó en 2010 Supernatural beings. From Slovenian myth and folktales y $A$ treasury of Slovenian Folklore. 101 Folktales from Slovenia (en colaboración con Z. Šmitek y R. Dapit). La primera incluye un glosario esloveno-inglés, articulado como diccionario de mitología local. La segunda fue elaborada con criterio antológico, que da cuenta de riqueza y variedad de tipos narrativos, e incluye 101 relatos de distintas regiones eslovenas, con metadatos, ilustraciones y comentarios explicativos, elaborados con criterios similares a los de la colección argentina de Chertudi. 
Para confrontar con colecciones eslovenas, se esboza en primer lugar un recorrido diacrónico por archivos y colecciones de narrativa tradicional argentina, en el que se señalan algunos puntos de contacto con los archivos eslovenos, para retomarlos luego en una comparación. ${ }^{8}$

\section{Colecciones generales de narrativa folklórica argentina}

La primera iniciativa sistemática de recolección de material folklórico argentino fue la Encuesta Folklórica de 1921, dirigida a maestros de escuelas públicas "Ley Láinez", convocados por el Consejo Nacional de Educación como intermediarios (e informantes), para recolectar y registrar por escrito dicho material de acuerdo con instrucciones institucionales. ${ }^{9}$ Según el instructivo, que fue el modelo al que los docentes debieron ceñirse, el Folklore comprende todos los productos culturales: artes, juegos, creencias, costumbres, ritos, técnicas, instrumentos, manufacturas y elementos decorativos, además de formas literarias en verso y prosa. Los «cuentos o ficciones» fueron subclasificados en el Instructivo, con criterio temático similar al de la clasificación universal de tipos folklóricos ${ }^{10}$ de Aarne-Thompson, en: 1. Maravillosos, 2. Religiosos y Morales, 3. Animistas o de espanto, 4. Humanos, 5. Animalísticos y 6. Gracejos verbales. Como otras especies literarias en prosa, el Instructivo menciona II Leyendas, III Casos, sucedidos, IV Tradiciones y V Mitos. La textura de los legajos manuscritos, producto de la Encuesta, presenta una reescritura corregida de la oralidad, con huellas de un enunciador plural. Convergen en ellos la voz institucional de los autores del instructivo, de maestros e «informantes» cuyos testimonios fueron transcriptos. Prevalece en el Instructivo la modalidad prescriptiva, con tendencia a descripción y ejemplificación. Los legajos, que se conservan en forma material y digitalizada en el Instituto Nacional de Antropología y Pensamiento Latinoamericano, sirvieron como base para antologías de relatos folklóricos en prosa de Chertudi (1960 y 1964), Giovannoni y Poduje (1988), y de folklore poético, como los Cantares Históricos de la Tradición Argentina de Fernández Latour de Botas (1962). Por la utilización del aula como ámbito de recolección, tales archivos son comparables a los de narrativa folklórica eslovena del maestro Dolenc.

Otro archivo de material folklórico, en verso, es el de Juan Alfonso Carrizo (nacido en 1895 y muerto en1957), quien combinó trabajo de campo con estudio erudito. Director del Instituto de la Tradición de la Provincia de Buenos Aires hasta 1949, recopiló en zonas rurales formas poéticas incluidas en habla, juegos, danzas y canciones, y rastreó su presencia en documentos europeos y americanos. Publicó Antiguos Cantos Populares Argentinos. Cancionero de Catamarca (1926), Cancionero Popular de Salta (1933), Cancionero Popular de Jujuy (1934), Cancionero Popular de Tucumán (1937), Cancionero Popular de La Rioja (1947) y Antecedentes Hispano-medievales de la Poesía Tradicional Argentina (1945). Por la investigación sistemática de poesía folklórica, su colección puede parangonarse con la de canciones eslovenas de Štrekelj, estudiada por Kropej Telban (2017).

La colección Cuentos Folklóricos de la Argentina. Primera y Segunda Series de Susana Chertudi (1960,1964), investigadora en Letras y Folklore, consta de dos volúmenes o "series", de 100 versiones cada uno. Cada Serie agrega una Introducción, Bibliografía, Léxico e Índice. Las versiones están acompañadas de metadatos -nombre, fecha y lugar de recolección-, y de una tabla de clasificación tipológica, realizada según índices de Tipos y Motivos Narrativos de Aarne-Thompson (1961), Thompson (1955-1958), Boggs (1930) y Hansen (1957). La autora selecciona la especie "cuentos", subdivididos en I. De Animales, II. Maravillosos, III. Religiosos, IV. Humanos y V. De fórmula. Organiza la colección con criterio antológico e incluye material de la Encuesta folklórica de 1921, de otros recolectores y de investigaciones de campo propias y de su esposo
8. Otra veta de este trabajo comparativo de narrativa folklórica argentina y eslovena es la confrontación de narraciones orales concretas. Tal veta fue desarrollada en un capítulo de autoría de quien esto escribe de la publicación conjunta realizada con las investigadores eslovenas (Palleiro comp. in press) y en una contribución presentada a la Conferencia Internacional "Narrative Culture in Local Places' celebrada en Slovenska Bistrica en junio 2019. En esta última oportunidad, quien escribe comparó versiones argentinas y eslovenas de la matriz folklórica "La dama fantasma"

(clasificada en el Índice de Motivos Folklóricos de Stith Thompson con el número $\mathrm{E}$ 332.3.3.1), ambientadas respectivamente en el cementerio de la Recoleta y en el Slovenska Bistrica castle. El resultado de esta comparación será volcado en un trabajo solicitado para su publicación en Studia Mythologica Slavica.

9. En este recorte, no se consideran los archivos anteriores a la Encuesta de 1921, ni los de viajeros y recolectores, que no poseen el grado de sistematicidad de las colecciones aquí tratadas. Por tratarse de un recorrido ceñido a la comparación con archivos eslovenos, tampoco se analizan las colecciones de narrativa indígena, estudiadas en trabajos anteriores (Palleiro, 2014).

10. El "tipo" es una combinación relativamente estable de unidades temáticas mínimas o "motivos", comunes a relatos folklóricos de distintos tiempos y lugares (Thompson, 1946). Tipos y motivos fueron codificados en índices como los que aquí se mencionan. 
11. Debe tenerse en cuenta que esta afirmación no es aplicable a la totalidad del material de la colección, a la que la autora fue agregando el fruto de nuevas recopilaciones. Tal es el caso, por ejemplo, de la versión 2627 del volumen IX; "Otro cuento de Ingale", recopilada de boca de Anunciado Caravetta, de 77 años, en Carmen de Patagones, Buenos Aires, en 1971 (Vidal de Battini; IX: 910-912).

12. Para una interesante reflexión sobre las variedades del español de la Argentina que incluye una aguda reflexión sobre esta obra de Vidal de Battini en relación con políticas lingüísticas, ver Kornfeld (2013).
Ricardo Nardi. Normaliza sintaxis y grafía según la norma-standard de escritura, aunque mantiene fenómenos fónicos como caída de consonantes intervocálicas y diptongaciones. Repone conectores y elimina reiteraciones e interjecciones, para mantener coherencia secuencial. Sobresalen la precisión clasificatoria ajustada a parámetros internacionales, la claridad expositiva de la Introducción y el rigor de selección bibliográfica. Los textos presentan indicios de reescritura de la oralidad, tales como marcas tipográficas en cursiva para señalar regionalismos. Por su precisión clasificatoria, la colección puede compararse con la de relatos folklóricos eslovenos de Kropej Telban, Šmitek y Dapit (2010).

Especial relevancia reviste la obra de Berta Vidal de Battini (nacida en 1900 y fallecida en 1984), cuyos Cuentos y Leyendas Populares de la Argentina (1980-1984, y 1995) constituyen el corpus más amplio de narrativa folklórica argentina, con versiones de todo el país en diez volúmenes. Incluye 3152 relatos, clasificados en "versiones" y "variantes" de "tipos" folklóricos. El registro narrativo, unido a la documentación de fenómenos lingüísticos, fue fruto de 30 años de trabajo y 150 viajes de campo auspiciados por el Consejo Nacional de Educación, que instó a maestros a colaborar con su trabajo (González y García, en prensa). El principal instrumento de recolección fue el cuestionario -cuyos modelos fueron recientemente publicados- que siguió la tradición de la Encuesta folklórica de 1921 (González et al., 2010). Debe tenerse en cuenta al respecto que la mayor parte del material de los cuentos y leyendas de la colección de Vidal de Battini fue recopilado entre 1940 y 1970 circa, años antes de su publicación (1980-1995). Dicho material procede, en líneas generales, de las Encuestas de 1949 y 1960, que agregaron al propósito de investigación lingüística y dialectológica de las variantes del español, el de la configuración de un corpus de narrativa folklórica de toda la Argentina. ${ }^{11}$ Vidal de Battini utilizó el material de las encuestas para dos trabajos diferentes, con un doble objetivo científico y didáctico, de difusión del acervo folklórico de toda la Argentina y de investigación sobre las variantes del español. ${ }^{12}$ En sintonía con el propósito de la Encuesta Folklórica de 1921, la obra de Vidal de Battini se inscribió también en un proyecto de política educativa y cultural tendiente a dar a conocer las diversidades regionales de la Argentina a través de su acervo narrativo folklórico. Un objetivo similar, si bien con mayor acento en las diversidades regionales que en la variación con respecto a tipos y motivos folklóricos universales como el principio que guió a la folklorista argentina, puede advertirse en la magna colección de narrativa folklórica eslovena coordinada por María Stanonik (1988-2020), que será comentada en una sección siguiente.

La colección de narrativa folklórica de Vidal de Battini comprendió dos especies narrativas, "Cuentos" y "leyendas", mientras que la de Chertudi solo incluyó "cuentos". Para su organización recurrió, al igual que Chertudi, a categorías temáticas de AarneThompson., y clasificó el material en 1) "Cuentos de animales" (volúmenes I, II y III), 2) "Maravillosos o de magia" (IV, V y VI), 3) "Humanos, morales y otros" (IX) ; "De personajes populares como "Pedro de Urdemales $(\mathrm{X})$ ", 4) "Acumulativos y encadenados" (X) y 5) "Cuentecillos y chistes $(\mathrm{X})$. El tomo $\mathrm{X}$, póstumo, que incluye "narrativa indígena", "cuentos de adivinanzas", "humanos y morales" y "varios", es el de mayor imprecisión clasificatoria. Para las leyendas (volúmenes VII y VIII), adoptó parámetros clasificatorios adecuados al corpus argentino, a saber: leyendas de 1) lugares; 2) piedras y cerros, 3) lagos y ríos, 4) ciudad perdida, 5) tapados, entierros o tesoros, 6) plantas, 7) animales y 8) cielo. Incluyó referencias cruzadas a la Clasificación Internacional de Leyendas establecida en Budapest (1963) por la convención de la International Society for Folk Narrative Research, que las divide en: I. Etiológicas y escatológicas, II. Históricas e histórico-culturales, III. Míticas y de seres y fuerzas sobrenaturales y IV. Religiosas (Vidal de Battini, VI: 9-10).. La obra está precedida por un "Prólogo", que explicita la metodología de recolección, en el marco de una investigación sobre habla regional. Pone el acento en la difusión geográfica de tipos y motivos, 
y agrega observaciones sobre lenguaje de los cuentos, rasgos fónicos del habla regional como seseo y cierre en un punto de vocales, e incluye consideraciones sobre "El estudio del cuento", desde la Antigüedad grecolatina con la fábula esópica, al Roman de Rénard francés. Cada grupo de relatos agrega una "Nota introductoria "con comentario temático general, clasificación por tipos y motivos de Antti Aarne y Stith Thompson (1928), Stith Thompson (1955-1958) y Ralph Boggs; observaciones sobre "variantes". Vidal de Battini incluye asimismo en su colección la cartografía de María Teresa Grondona que señala la distribución de versiones en mapas del territorio argentino, según parámetros del método histórico-geográfico. Incorpora metadatos como fecha y lugar de recolección, nombre, edad e instrucción del "informante" y observaciones esporádicas sobre estilo. En su confrontación con la de Chertudi, esta colección es mucho más amplia y menos precisa en la aplicación de parámetros clasificatorios similares para la organización del material con un criterio antológico, propio de un paradigma coleccionista. Al igual que Stanonik en su "Glosario", agrega explicaciones sobre el significado de lexemas indígenas y regionalismos. Como rasgo distintivo, sobresale la vastedad del material, que incluye resultados de investigaciones de campo propias y de recolectores como los maestros, a quienes, bajo la forma de encuestas, encomendó la recopilación y registro a lo largo y ancho del país, con método similar al de la Encuesta Folklórica de $1921 .{ }^{13}$ Del mismo modo que en la colección de María Stanonik (1988-2020), prima el criterio antológico sobre el interpretativo, con el afán de ofrecer una colección documental de cuentos y leyendas argentinas (Vidal de Battini, X, 1995: 7).

El equipo coordinado por Aída González, del Instituto de Investigaciones Lingüísticas y Filológicas "Manuel Alvar" (Universidad Nacional de San Juan) donde se domicilia el FONVIBA, contribuyó a esta investigación, de alcances más amplios de los que se da cuenta en este artículo, centrado en el recorrido diacrónico de las colecciones argentinas, para el trazado de lineamientos generales de comparación con colecciones eslovena. Proporcionó así datos sobre el contenido del FONVIBA, difundido también en obras como el Atlas Lingüístico de Cuyo, - referido al léxico del agua, la vid, el vino y el carneo del cerdo- (González et al., 2018)-. La descripción y comentario de estas piezas es el eje de una contribución realizada por la lingüista en colaboración con la investigadora Graciela N. García, incluida en un volumen en prensa, orientado a dar cuenta de los resultados finales del proyecto (González y García, en prensa). Las piezas del FONVIBA comprenden 180.000 legajos con cuestionarios manuscritos confeccionados -al igual que la Encuesta de 1921- por maestros de escuelas "Ley Láinez", entre 1946 y 1960. Contiene también 125.000 proverbios y frases de interés lexicográfico que configuran un corpus de 63.000 palabras, un archivo fotográfico, uno sonoro de registros grabados y el epistolario personal de esta investigadora.

De las colecciones regionales cabe considerar Cuentos populares de La Rioja de Juan Agüero Vera (1965) y Cuentos y leyendas de La Pampa (1988) de Nélida Giovannoni y María Inés Poduje (1988). ${ }^{14}$ Juan Zacarías Agüero Vera fue un investigador regional, en contacto con los máximos expertos en Folklore del momento, Augusto Cortazar y Susana Chertudi, autores de prólogo y clasificación de relatos, respectivamente. La recopilación, realizada con criterio antológico según pautas explicitadas en el estudio introductorio de Cortazar, "El método histórico-geográfico y el estudio de los cuentos populares", contiene 37 "cuentos" y "leyendas" -con predominio cuantitativo de los "cuentos maravillosos". En sus "Notas", Chertudi ordena el material según Índices de Tipos y Motivos de Antti Aarne y Stith Thompson (1961), Stith Thompson (1955-1958), Terence Hansen (1957) y Ralph Boggs (1930). La obra incluye un "Prólogo del autor" de tono lírico, que alude a la recolección "de boca de ancianos iletrados [...] buscando [...] el raudal incontaminado de la verdadera tradición" (Agüero Vera, 1965: 44). Grafía y sintaxis están normalizadas para adecuar los textos al canon escritural
13. Cabe destacar que en los archivos del Fondo "Vidal de Battini” de la Universidad Nacional de San Juan de las Encuestas de 1949 y 1960 , las páginas referidas a "cuentos" aparecen cuidadosamente arrancadas, como se pudo comprobar en un trabajo de investigación realizado in situ por quien esto escribe. Un ejemplo de que estos datos estaban presentes en las Encuestas originales es el de un folio archivado en el INILFI en una caja con el rótulo de "Sueltos", sin fecha precisa. Este folio incluye el cuento "El hombre con piel de oso" enviado por la maestra Mercedes A. de Godoy sine data (presumiblemente correspondiente a la Encuesta de 1960, ya que otros legajos con información dialectológica incluidos en la misma caja tenían esa fecha). Esta maestra desempeñaba funciones en la Escuela No. 163 de Valle Hermoso, Vinchina, departamento General Sarmiento, La Rioja, en donde recolectó el relato. La falta de cuentos en los legajos de Vidal de Battini puede deberse a múltiples razones. Al servirse de una misma Encuesta para dos trabajos, diferentes -la investigación dialectológica cuyos resultados fueron sistematizados en El español de la Argentina y la investigación en narrativa folklórica publicada en Cuentos y leyendas populares de la Argentina- la ilustre folklorista pudo haber arrancado las páginas correspondientes para archivarlas por separado en carpetas o legajos que no hayan llegado a manos de quienes custodian el FONVIBA. Esta falta de legajos correspondiente a los cuentos de Vidal de Battini ha sido constatada también por Aída González, quien prestó a quien escribe su valiosa colaboración en la tarea de revisión de los legajos de las encuestas. Las causas de esta ausencia de narraciones en los legajos de Vidal de Battini quedará irremediablemente en el terreno de las conjeturas por el fallecimiento de la autora, pero tal ausencia no invalida en absoluto la credibilidad de las fuentes utilizadas para la magna recopilación de cuentos, sino que, simplemente, impide a los investigadores el cotejo de los legajos originales con el material publicado.

14. Por razones de espacio, se omiten referencias a colecciones como "Cuentos de la Tradición Oral Argentina” de Jesús María Carrizo y Guillermo Perkins Hidalgo incluidos en la Revista del Instituto Nacional de la Tradición (1948), y a colecciones de narrativa indígena, en este recorrido orientado a la comparación con archivos eslovenos. Para un estudio de las colecciones de narrativa indígena, ver Palleiro (2014). 
Los Cuentos y leyendas de La Pampa (1988) de la Licenciada en Letras Nélida Giovannoni y la antropóloga María Poduje, investigadora de la Dirección General de Cultura de La Pampa, utilizan categorías temáticas de los Índices de Aarne-Thompson y Thompson para los grupos de versiones, pero no para cada relato individual. Adoptan también parámetros de Vidal de Battini, para clasificar "cuentos" y "leyendas". Incluyen 69 cuentos de animales, 22 humanos, 22 maravillosos, 5 religiosos; 24 leyendas religiosas y etiológicas y 60 leyendas de creencia. Incorporan material de colecciones anteriores como la Encuesta Folklórica de 1921, Chertudi, Vidal de Battini, de recolectores regionales y de investigaciones de campo. Agregan "Notas", "Bibliografía" y una "Introducción" que da cuenta del estado del arte de la narrativa pampeana en el momento de edición de esta obra en la que la precisión clasificatoria es reemplazada por criterios más flexibles.

Por su criterio antológico y clasificatorio, las colecciones de Agüero Vera y de Giovannoni-Poduje guardan relación con la de relatos eslovenos de Kropej Telban, Šmitek y Dapit (2010).

Un cambio de modalidades de archivo se advierte en Estructura del miedo de la antropóloga Martha Blache (1991[1982]), que incorpora la dimensión analítica. La obra, fruto de su Tesis de Doctorado en Folklore de la Indiana University, comprende un corpus de leyendas recogidas entre migrantes paraguayos en Buenos Aires, en 19721974. Este material sirve como pre-texto para analizar representaciones culturales del grupo, con enfoques estructurales y contextualistas de las "Nuevas Perspectivas del Folklore". Relatos sobre seres mitológicos como la Pora o el Luisón son considerados como signos identitarios de una comunidad migrante. Esta obra marca un viraje en la modalidad de archivo, que privilegiaba hasta entonces criterios antológicos. La orientación contextualista se abre al abordaje semiótico de la narrativa tradicional, como vía de acceso al universo de valores del grupo. El estudio de criaturas míticas como las arriba mencionadas guarda semejanza con el de Ivančič Kutin sobre las Krivopete, símbolos identitarios de comunidades alpinas.

Colecciones más recientes como Cuentos de las tres abuelas de Silvia García y Diana Rolandi (2000) presentan distintas etapas de formación de un archivo, constituido por materiales de campo recolectados en 1995 en Antofagasta de la Sierra, Catamarca, en un proyecto UNESCO de promoción del patrimonio cultural inmaterial. Las autoras, investigadoras del Instituto Nacional de Antropología y Pensamiento Latinoamericano, dan cuenta de una reescritura progresiva en clave de género, en la que gravita lo cotidiano. Abre la obra una "historia" pretextual de"tres abuelas", luego reescrita en una colección de textos clasificada, según parámetros tipológicos, en "cuentos maravillosos", "de animales" y "humanos", con agregado de categorías vernáculas como "la Pacha". Las categorías de índices temáticos, privilegiadas por Chertudi y Vidal de Battini, sirven aquí para la reelaboración ficcional del material narrativo. Esta reescritura polifónica de relatos orales combina el documento antropológico con la elaboración literaria. Por su carácter de promoción del patrimonio intangible, puede parangonarse con la publicación Zbirke povezukejo-Le collezioni uniscono de promoción del patrimonio de comunidades fronterizas ítalo-eslovenas.

Otro conjunto de archivos de narrativa folklórica es el de María Inés Palleiro, que pone énfasis en procedimientos de ficcionalización del contexto, aunque hay entre ellos diversidad de criterios, por condicionamientos editoriales. Por razones de espacio, se mencionarán solo archivos éditos en formato libro, con exclusión de publicaciones colectivas. Las cuatro colecciones de narrativa folklórica de Palleiro: 1) "El escondite mágico" yotros cuentos folklóricos riojanos (1990), 2) "Los tres pelos del diablo". Cuentos maravillosos de la cultura popular argentina (1992), 3) "La 
fiesta en el cielo". Cuentos populares de animales (1998) y 4) "La fuga mágica": Cuentos maravillosos de la provincia de La Rioja, República Argentina (2011), privilegian la dimensión antológica, mientras que los trabajos que privilegian el análisis son: 1) Estudios de Narrativa Folklórica (1990), 2) Nuevos Estudios de Narrativa Folklórica (1992), 3) Fue una historia real. Itinerarios de un archivo (2004), 5) El cuento folklórico riojano: una introducción a la narrativa oral (2016), 6) Discursos de migración, desarraigo y exilio en el Cono Sur: entre la oralidad y la escritura (2017) y 7) La dama fantasma. Los laberintos de la memoria en el relato folklórico (2018). En las antologías, la autora adecuó transcripciones de relatos orales al canon escritural y los clasificó según índices temáticos de Antti Aarne y Stith Thompson, Hans Uther; Ralph Boggs, Terence Hansen, e Índice de Motivos de Stith Thompson. Eligió para cada colección relatos representativos de distintos tipos narrativos, para evidenciar transformaciones contextuales. Incluyó la dimensión del análisis en estudios introductorios, y agregó tablas de narradores, clasificaciones y notas en las que destacó estilo, composición y performance de narradores. Individualizó también procedimientos discursivos de ficcionalización del contexto, con aportes de genética textual, teoría del archivo y teoría informática del hipertexto. En las obras analíticas, Palleiro privilegió la reflexión teórica para el estudio discursivo de relatos recogidos en investigaciones de campo, registrados con grafía intermedia entre oralidad y escritura, y agregó bibliografía internacional sobre narrativa folklórica. Su propósito fue proporcionar, además del registro, una aproximación a procesos de textualización del contexto, desde una perspectiva semiótica. La autora subrayó asimismo la relevancia de la corporalidad del narrador, y destacó la incidencia de creencias colectivas en transformaciones contextuales del relato folklórico, considerado como expresión estética secuencial de identidades sociales, con sello individual del narrador. Diseñó de este modo una metodología de estudio de narrativa oral y eventuales fijaciones escriturarias centrada en itinerarios de dispersión de «matrices», a las que definió como conjuntos de núcleos temáticos, compositivos y estilísticos almacenados en la memoria de los narradores. El concepto de «matriz» agrega a regularidades temáticas de índices universales, aspectos de composición y estilo -destacados por Bajtín en su caracterización de los géneros discursivos-. Consideró la narración como principio de organización secuencial de la experiencia, recreada en un universo ficcional (Bruner, 2003), con potencialidad deconstructiva en itinerarios múltiples semejantes a los del hipertexto virtual, que reproduce el recorrido dispersivo del recuerdo. La matriz, identificada mediante confrontación de relatos, sirve, según Palleiro, como pretexto de textualizaciones virtualmente infinitas en contextos diferentes (Palleiro, 2004, 2016, 2018 y 2019a). En un capítulo individual de una obra colectiva, la autora incluyó, además de relatos folklóricos, testimonios personales sobre experiencias migratorias, producto de una investigación sobre narrativa de desarraigo que consideró la problemática de los desaparecidos (Palleiro, 2017b: 65-87).

Por la modalidad de ordenamiento y clasificación tipológica, las antologías de Palleiro guardan semejanza con la obra de Kropej Telban Šmitek y Dapit, A treasury of Slovenian Folklore. 101 Folktales from Slovenia, mientras que su obra teórica, que reflexiona sobre paradigmas del Folklore, tiene contacto con Le Folklore littéraire, de Stanonik.

La aproximación a colecciones argentinas, orientada a la confrontación con archivos eslovenos, tuvo como base un recorte con punto de partida en la Encuesta folklórica de 1921. Los criterios de registro y clasificación se extendieron desde el ordenamiento según índices temáticos de Tipos y Motivos hasta la reescritura ficcional de registros orales. Hay una conexión intertextual entre colecciones y semejanzas con archivos eslovenos. Esto evidencia paradigmas y respuestas similares frente a problemas clasificatorios de narrativa folklórica, en distintos puntos del planeta. 
15. Para más datos sobre esta problemática, ver Palleiro (comp.), 2017: 19-24 y 65-87.

\section{Archivos folklóricos argentinos y eslovenos: hacia una aproxima- ción comparativa}

Este recorrido diacrónico evidenció líneas de contacto con archivos eslovenos. La Encuesta folklórica de 1921 es comparable con el archivo esloveno de Baudouin de Courtenay (1845-1929), quien lanzó una invitación a recopilar material folklórico en periódicos a partir de instrucciones similares a los recolectores. La invitación estuvo especialmente orientada, al igual que las Encuestas de Vidal de Battini, a recopilar material dialectológico. También el archivo de Dolenc, docente esloveno que recolectó material folklórico entre sus alumnos, presenta elementos comunes con la Encuesta folklórica de 1921, por tratarse de registros manuscritos recopilados en ámbito escolar. Dicha Encuesta respondió sin embargo a un proyecto institucional desarrollado en toda la Argentina en un único momento, mientras que el archivo Dolenc nació de la iniciativa individual de un solo docente en una región, sostenida por veinte años. La obra de Gašper Križnik (1849-1904), primer recolector de material narrativo folklórico en formas dialectales, es comparable con la de recolectores regionales argentinos como Agüero Vera, Giovannoni-Poduje y con la colección de relatos riojanos de Palleiro (1990a, 2016), mientras que el cancionero folklórico de Štrekelj (1895-1923) puede parangonarse, por su sistematicidad, con los Cancioneros de J. A. Carrizo (1926-1947).

La obra de Milko Maticetov, de los años 50, propone una aproximación comparativa con el folklore italiano en zonas fronterizas, continuada actualmente en el Proyecto Zbirke povezukejo-Le collezioni uniscono, sistematizado por Roberto Dapit, Barbara Ivančič Kutin y Špela Ledinek-Lozej (2015). Algunos de sus registros pueden compararse con el material argentino de zonas de cruces culturales estudiado en Palleiro (2017) que incluye, además de relatos tradicionales, narraciones de experiencias de desarraigo vinculadas con la problemática intercultural. ${ }^{15}$ La publicación ítalo-eslovena Zbirke povezukejo-Le collezioni uniscono, que recoge manifestaciones del patrimonio cultural de comunidades fronterizas alpinas, guarda semejanza con la de García y Rolandi, encuadrada en un proyecto de valorización patrimonial de relatos de la región cordillerana de Antofagasta de la Sierra.

Especial interés comparativo reviste el magno corpus de narrativa folklórica de Vidal de Battini, cuya orientación antológica resulta similar a la de colección Glasovi (Voices) dirigida por Stanonik. Tal similitud puede extenderse a los perfiles de ambas investigadoras. Vidal de Battini (1900-1984) se graduó en Lengua y Literatura en 1923 en la Universidad de Buenos Aires, donde obtuvo luego su doctorado. Docente e investigadora en Folklore, Lingüística y Filología, fue además Inspectora de Escuelas del Ministerio de Educación de la Nación (Palleiro 2015: 1843-1845). También Stanonik tuvo formación en literatura, inclinada a la reflexión teórica. Fue docente universitaria de Folklore y Literatura en la Universidad de Ljubljana, como Vidal de Battini, quien enseñó Filología Hispánica y Folklore en la Universidad de Buenos Aires, donde fue jefa de la sección Folklore del Instituto de Ciencias Antropológicas. Fue elegida académica de número de la Academia Argentina de Letras en 1983, como también lo fue Stanonik en su país. Al igual que Vidal de Battini, Stanonik vinculó los estudios folklóricos con disciplinas lingüísticas y literarias, y las directrices de su pensamiento, sintetizadas en Le folklore littéraire. Approche pluridisciplinaire d un phénomène syncrétique (2009), destacan la vinculación entre Folklore, Teoría Literaria y Lingüística, especialmente en el estudio de variantes dialectales. También Vidal de Battini estudió variantes dialectales con el método de la encuesta, que le brindó material para los Cuentos y leyendas populares de la Argentina. En El Habla Rural de San Luis (1949) analizó variantes regionales del español conectadas con cambios culturales, y en El Español de la Argentina (1964) amplió dicho análisis a variantes dialectales del español argentino. Al igual que Stanonik, enriqueció el Folklore con una visión integradora del lenguaje y la cultura tradicional. 
El rasgo distintivo de la colección de Vidal de Battini es la extensión cuantitativa, que incluye material de investigación de campo propia y de otros recolectores a quienes encomendó esta tarea a lo largo y ancho del país, facilitada por su cargo de Inspectora General de Escuelas. También Stanonik, además de recopilar ella misma, encomendó la recolección a expertos de distintas regiones, pero los puso además al cuidado de ediciones, lo que le permitió publicar más de 50 volúmenes. La colección de Vidal de Battini agrega notas al pie con explicaciones sobre lexemas indígenas y regionalismos, y también Stanonik incluye en cada tomo un glosario de voces dialectales. Del mismo modo que en Stanonik, prima en Vidal de Battini el criterio antológico sobre el interpretativo, para ofrecer una colección documental. Al igual que los de Vidal de Battini, los volúmenes de Stanonik están acompañados de mapas de localización regional de cuentos, leyendas y otras especies narrativas, pero, a diferencia de ella, muchos volúmenes están acompañados de ilustraciones de los mismos narradores. La presentación de cada tipo de Vidal de Battini agrega, al igual que Stanonik en cada volumen, observaciones sobre cultura local, fauna y creencias colectivas. Su apertura hacia variantes contextuales introduce un quiebre con respecto al paradigma tipificante, que concuerda con su interés por el habla regional dentro del sistema general de la lengua. El paradigma de Stanonik es también abierto, influenciado por dialectología y folklore literario. Su obra teórica evidencia conocimiento de estudios de Folklorística a nivel mundial, reflejado en la agudeza para seleccionar compiladores y prologuistas. Ambas investigadoras recolectaron un magno corpus de narrativa folklórica de un país, con mapeo de relatos. Hay, sin embargo, entre ellas una diferencia fundamental. Mientras que Vidal de Battini, en su rol de inspectora de escuelas, recibió material de docentes, estos no fueron mencionados como recopiladores, sino que la totalidad del material narrativo aparece enteramente a su cargo, al haber realizado ella la tarea de sistematización. ${ }^{16}$ Por el contrario, Stanonik, como coordinadora, supervisó una tarea de conjunto, y cada volumen tiene reconocimiento de autoría de un compilador regional. Esto le permitió no solo ampliar el espectro de obras editadas, sino también formar equipos de folklorólogos en toda Eslovenia. Ambas colecciones incluyen material folklórico de jóvenes países, cuyo estudio comparativo resulta fecundo para ubicar producciones nacionales en un mapa mundial.

La clasificación por tipos de Vidal de Battini resulta semejante a la del índice tipológico de Kropej Telban, quien incorpora un relato para cada tipo, con criterio antológico.

Por su parte, Kropej Telban (en Palleiro, in press) encontró similitudes entre la colección de Vidal de Battini y la de Štrekelj., quien introdujo por vez primera el término "Folklore" en los estudios humanísticos eslovenos y que, al igual que Vidal de Battini, centró su atención en habla dialectal y gramática histórica. Si bien Štrekelj llegó a publicar una edición sistemática de canciones folklóricas eslovenas, su archivo manuscrito de 1154 relatos folklóricos, enviados por recolectores de distintas regiones, a diferencia del de Vidal de Battini, permaneció inédito, por su temprana muerte. Según afirma Kropej Telban, aunque comparada con las colecciones de los Grimm (1857) y Afanasjev (1957) la calidad de los materiales es heterogénea, su legado es de gran relevancia para la cultura eslovena, al igual que el de Vidal de Battini para la cultura argentina. Si bien la investigadora eslovena comparó este archivo con el de Vidal de Battini, cabría tal vez compararlo también, según se ha anticipado, con la obra de Carrizo (1895-1957), recolector del mayor corpus de canciones tradicionales argentinas. La obra de Štrekelj puede también ser comparada con la de Isabel Aretz (1913-2005), quien, además de antologías didácticas, publicó El folklore musical argentino (1952), Música tradicional argentina. Tucumán, y su tesis de doctorado sobre Música tradicional de La Rioja.

La orientación antológica de Chertudi y Vidal de Battini guarda también semejanza con la antología de narrativa folklórica eslovena de Kropej Telban et al. (2010). Como
16. La misma Vidal de Battini reconoció la colaboración de los maestros en su tarea de recolección, favorecida por su rol de Inspectora General de Escuelas. Ella misma solicitó, en un apartado especial de las Encuestas, que los maestros transcribieran relatos narrados por informantes locales. En las Instrucciones para la Encuesta sobre el Habla Regional enviadas en 1949 a las Escuelas Primarias por el Consejo Nacional de Educación -recientemente publicadas- figura en efecto el apartado "Las narraciones". A modo de ejemplo, puede citarse el texto de uno de estos cuestionarios, que indica con precisión: “Envíe, por separado, una nómina de las personas del lugar que sepan cuentos y leyendas populares (...) Envíe todas las variantes que pueda recoger, transcriptas fielmente por el narrador y los datos de éste" [sic. El subrayado es de quien escribe] (González et al. 2010: 284). Por otra parte, en la “Introducción" del primer volumen de los Cuentos y leyendas populares de la Argentina, la misma Vidal de Battini reconoce que: “El Consejo Nacional de Educación determinó que los maestros primarios me apoyaran con su colaboración en todo el territorio" (Vidal de Battini, I: 14). Sin embargo, ellos no fueron mencionados con nombre y apellido en ninguno de los paratextos de la obra, como sí lo hicieron, por ejemplo, Giovannoni y Poduje al referirse a los legajos de la Encuesta Folklórica de 1921. Es así como las autoras especifican que una de las versiones del relato el relato "La zorra y la perdiz" fue recolectada por el "maestro Luis Funes" en Ceballos, La Pampa (Giovannoni y Poduje, 1988: 66) Las investigadoras pampeanas diferencian estos registros de aquellos recolectados por ellas mismas, como por ejemplo, la versión de "El zorro y la perdiz" narrada por María Peralta, que fue registrada en Santa Rosa, el 27 de agosto de 1986 por Giovannoni y Poduje (1986: 67). El registro de transcripciones realizadas por los maestros, en su rol de recolectores e intermediarios, se advierte en aquellas colecciones que tomaron como modelo cuestionarios y Encuestas folklóricas, o que incorporaron fuentes procedentes de dichas Encuestas. Este es el caso de las recolectoras antes nombradas, $y$ el de Chertudi quien, por ejemplo, en la versión No. 12 de su Segunda Serie, correspondiente al relato "El zorro aprende a cantar" consigna, junto con los datos del informante losé Kolian, el lugar de recolección, Jacinto Aráuz, Pampa Central, y el nombre de la recolectora, la directora de escuela Magdalena Bayo, de la Escuela No. 33, con la cita de fuente, correspondiente al legajo No. 7, página 28, de la Colección de Folklore de la Encuesta folklórica de 1921 (Chertudi 1964: 38). Las demás colecciones aquí estudiadas no recurrieron a encuestas ni a cuestionarios para recolectar el material. 
17. Para su índice, Kropej Telban (2015) tomó como base el sistema clasificatorio del finés Aarne, revisado en 1928 por el norteamericano

Thompson -también autor del Motif Index of Folk Literature-. El Tale Type Index de Aarne-Thompson, que es aún hoy modelo canónico de clasificación, fue actualizado en 2004 y 2011 por el alemán Uther, y citado desde entonces mediante el acrónimo ATU. Kropej Telban identificó 151 tipos en el Type index of Slovenian folktales, animal tales and fables, y agregó 17 tipos vernáculos, con lo cual dio pie para una apertura del sistema internacional a categorías de otras latitudes. Ciertamente, entre los Índices Tipológicos recientes, el de Kropej Telban y el de relatos gallegos de Noia Campos (2010), que agregan un ejemplo textual para cada tipo, fueron elaborados con criterios abiertos capaces de despertar interés del lector curioso por temas y estilos de narrativa folklórica local.

Esto los convierten en ejemplos a imitar para la elaboración de un catálogo tipológico argentino.
Kropej Telban, también Chertudi restringió el número de textos para privilegiar exactitud en la identificación de tipos en colecciones acotadas, adecuadas a parámetros tipológicos internacionales.

Tal como se afirmó más arriba, la dimensión analítica de un archivo sobre seres míticos como Krivopete de Ivančič Kutin puede parangonarse con Estructura del miedo de Blache, en la que criaturas míticas como la Pora son considerados como signos identitarios de una comunidad. Asimismo, la citada antología de Kropej Telban et al., que ofrece ejemplos de tipos y motivos del folklore literario esloveno, guarda semejanza con las antologías de narrativa folklórica de Palleiro, que presentan una muestra de tipos de cuentos animalísticos y maravillosos argentinos. La reflexión teórica incluida en sus estudios puede compararse con la de Stanonik sobre el folklore literario, si bien los trabajos de Palleiro tienen como anclaje un corpus narrativo, mientras que Stanonik ofrece una comparación de orientaciones teóricas de Folklorística en distintos puntos del globo. Esta visión universalista de Stanonik sirve como inspiración para futuras investigaciones, direccionadas a situar los estudios de narrativa folklórica argentina en el contexto mundial. Merece recordarse también al respecto que Kropej Telban es autora del Índice Tipológico de Narrativa Folklórica Eslovena (en idioma vernáculo), del que ha sido ya publicado el tomo de Cuentos de Animales. Este volumen sirve como modelo para un Catálogo Tipológico de Narrativa Folklórica Argentina, en el que la clasificación por tipos y motivos se pueda ver enriquecida por aspectos compositivos y estilísticos incluidos en el concepto de "matriz". ${ }^{17}$

Cabe destacar por último, en relación con el impacto del proyecto, la visita de Stanonik e Ivančič Kutin a Buenos Aires, en 2018 y 2019, respectivamente, para recoger testimonios de narrativa folklórica de migrantes eslovenos en la Argentina que, una vez procesados, enriquecerán la colección Glasovi. Archivos como la Encuesta folklórica de 1921 y el de Vidal de Battini, sustentados por paradigmas coleccionistas, respondieron al objetivo político de reconstruir una tradición argentina a partir de culturas hispánica y criolla, como defensa frente a la amenaza cultural del flujo inmigratorio. Por esta razón, no recogieron relatos de migrantes, cuya incorporación es objetivo de la colección de Stanonik. Testimonios orales de migración y exilio que recurren a matrices folklóricas fueron incluidos en una obra que destaca la eficacia del relato folklórico para relatar experiencias de desarraigo (Palleiro comp., 2017). Colaboraron en ella Lucila Pagliai -quien estudió aspectos histórico-culturales de migración europea en la Argentina-, junto con Rosa Grillo y Valentina Ripa de la Universitá di Salerno. Recientemente, se han recopilado también testimonios de desaparecidos durante la última dictadura militar argentina, en forma de relatos orales y virtuales de quienes compartieron las aulas del Colegio Nacional de Buenos Aires entre 1971 y 1976 (Palleiro comp. 2019b). El propósito de esta obra fue incorporar relatos orales de migrantes -como los que proyecta incluir Stanonik en su colección de narrativa eslovena-y de experiencias de exilio, a los archivos de la memoria social argentina.

En síntesis, en este recorrido cronológico tendiente a una aproximación comparativa a las colecciones y archivos argentinos y eslovenos, puede trazarse, en el contexto argentino, una línea de continuidad entre la Encuesta folklórica de 1921, la colección monumental de Vidal de Battini (1980-1995) y la antología de Giovannoni y Poduje (1988). El común denominador de estas colecciones tiene que ver con un propósito educativo, reflejado en una tarea de divulgación del acervo narrativo folklórico de distintas regiones argentinas a lo largo y a lo ancho de todo el territorio, o de una provincia en particular. Este propósito es similar al que animó, en el contexto esloveno, a la recolección al maestro Dolenc (1956-1990), y, en época más reciente, a Stanonik (1988-2020), con su colección monumental que incluye material narrativo folklórico de las distintas regiones de Eslovenia. Por su parte, la colección regional de Agüero Vera (1965) guarda, por un lado, una estrecha relación con la de Chertudi, quien se 
encargó de clasificar por tipos y motivos los relatos. Por otro, su propósito de reunir en forma antológica la cuentística regional con un fin documental se inscribe en una orientación delineada por Juan A. Carrizo, quien recopiló material folklórico en verso de distintas provincias argentinas, del mismo modo que lo hizo Karel Štrekelj en el ámbito esloveno. La minuciosidad clasificatoria de Chertudi (1960-1964) permite situarla dentro de la línea de trabajo que, en el ámbito esloveno, desarrollaron, en las últimas décadas Dapit, Šmitek y Kropej Telban (2010). Estas obras y autores, que privilegiaron el criterio antológico, se inscriben a su vez dentro de un paradigma coleccionista del Folklore. En el viraje hacia un paradigma comunicativo y hacia nuevas modalidades de archivo que incluyen la dimensión interpretativa y la puesta en texto de las operaciones de reescritura se ubican las colecciones de Blache (1991[1982]), García y Rolandi (2000) y Palleiro (1990 y 2017). Tal modalidad centrada en la dimensión interpretativa es similar a la adoptada, en el ámbito esloveno, en las contribuciones más recientes de Ivančič Kutin.

Estas semejanzas y diferencias revelan la productividad de comparar archivos argentinos y eslovenos, destacada por Tjaša Lorbek, lectora de esloveno de la Universidad de Buenos Aires (Lorbek en Palleiro, in press). El recorrido por textos y colecciones de ambas culturas refuerza el concepto de identidad diferencial que, como afirma Bauman (1972), constituye la base social del Folklore.

\section{A modo de cierre}

La comparación de archivos argentinos y eslovenos es veta fecunda para el estudio de la diversidad cultural. Las colecciones analizadas tuvieron en común su conexión con el Folklore, campo disciplinar relacionado con expresiones estéticas de identidades sociales, sustentado por paradigmas que van del coleccionismo a enfoques contextualistas. Según Derrida (1997), el archivo es un principio de ordenamiento y domiciliación de la memoria. El Institute of Ethnology de la Slovenian Academy of Social Sciences sirve de domiciliación física y simbólica de colecciones eslovenas, del mismo modo que el Instituto Nacional de Antropología y Pensamiento Latinoamericano domicilia la Encuesta folklórica de 1921, y el Instituto de Investigaciones Lingüísticas y Filológicas "Manuel Alvar" alberga el Fondo Vidal de Battini. Cada colección de relatos folklóricos es también, en sí misma, un "archivo de la memoria" (Barrenechea, 1993: 7).

Este recorrido por relatos y colecciones intentó destacar el valor de los archivos del Folklore para configurar memorias sociales, no solo mediante la preservación patrimonial del pasado, sino también por su actualización en el presente. Desde esta óptica, la tradición custodiada en archivos es fuerza transformadora, capaz de resignificar identidades y promover cambios sociales. ${ }^{18}$ Su sistematización y difusión, acompañada de reflexiones sobre paradigmas que los sustentan, estimula el diálogo entre culturas, indispensable para situar expresiones identitarias locales en un mapa global.
18. Estos archivos pueden servir para mejorar condiciones de vida de comunidades locales mediante actividades de transferencia como el turismo (Palleiro y Peltzer, en prensa) además de constituir herramientas de educación a la diversidad, en el marco de la recientemente sancionada ley de enseñanza obligatoria del Folklore en las escuelas. 


\section{Q Bibliografía}

》Aarne, A. \& S. Thompson (1961 [1928]). The types of the folktale: a classification and bibliography. Helsinki: Academia Scientiarum Fennica.

"Afanasjev, A. (1957). Narodnye russkie skazki (Russian Folktales). Moskva: V. Ja. Propp.

" Agüero Vera, J. (1965). Cuentos populares de La Rioja. La Rioja: Imprenta del Estado y Boletín Oficial.

»Aretz, I. (1946). Música tradicional argentina: Tucumán, historia y folklore. San Miguel: Universidad Nacional de Tucumán.

»Aretz, I. (1952). El folklore musical argentino. Buenos Aires: Ricordi.

"Aretz, I. (1978). Música Tradicional de La Rioja. Caracas: Inidef.

»Bajtín, M. (2002). Estética de la creación verbal. Buenos Aires: Siglo XXI.

»Barrenechea, A.M. (2003). Archivos de la memoria. Rosario: Beatriz Viterbo.

»Baudouin de Courtenay, J. (1910). A Baudouin de Courtenay Anthology. Bloomington: Indiana University Press.

" Bauman, R. (1972). "Differential identity and the social base of Folklore". En: Paredes, A. et al. (eds.), Toward new perspectives in Folklore. Austin and London: University of Texas Press, 31-41.

» Blache, M. (1991 [1982]). Estructura del miedo. Narrativas folklóricas guaraníticas. Buenos Aires: Plus Ultra.

»Boggs, R. (1993 [1930]). Index ofSpanishfolktales. Helsinki: Academia Scientiarum Fennica.

» Bruner, J. (2003). La fábrica de historias. Derecho, literatura, vida. Buenos Aires: Fondo de Cultura Económica.

»Carrizo, J. A. (1926). Antiguos cantos populares argentinos. Cancionero de Catamarca. Buenos Aires: Silla.

»Carrizo, J. A. (1933). Cancionero popular de Salta. Buenos Aires: Baiocco.

»Carrizo, J. A. (1934). Cancionero popular de Jujuy. Tucumán: Miguel Violento.

" Carrizo, J. A. (1937). Cancionero popular de Tucumán. Buenos Aires: Baiocco.

"Carrizo, J. A. (1945). Antecedentes hispanomedievales de la poesía tradicional argentina. Buenos Aires: Publicaciones de Estudios Hispánicos.

»Carrizo, J. A. (1947). Cancionero popular de La Rioja. Buenos Aires: Baiocco.

»Carrizo, J. M. y G. Perkins Hidalgo (1948). "Cuentos de la Tradición Oral Argentina”. Revista del Instituto Nacional de la Tradición, I, 209-257.

"Chamosa, O. (2010) The Argentine Folklore Movement: Sugar Elites, Criollo Workers, and the Politics of Cultural Nationalism, 1900-1955. Tucson: University of Arizona Press.

"Chamosa, O. (2012) Breve historia del folclore argentino (1920-1970). Identidad, política y nación. Buenos Aires: Edhasa.

»Chein, D. (2011). “Argentinos de profesión. El debate nativista en torno a la poesía gauchesca”. Revista de Crítica Literaria Latinoamericana, XXXVII, 25-48. 
»Chertudi, S. (1960). Cuentos folklóricos de la Argentina. Primera Serie. Buenos Aires: Instituto Nacional de Filología y Folklore.

»Chertudi, S. (1964). Cuentos folklóricos de la Argentina. Segunda Serie. Buenos Aires: Instituto Nacional de Antropología.

"Consejo Nacional de Educación. (1921). Colección de Folklore. Encuesta foIklórica del Consejo Nacional de Educación a los maestros de las escuelas Ley Láinez. Buenos Aires: Hemeroteca del Instituto Nacional de Antropología y Pensamiento Latinoamericano.

»Dapit, R.; B. Ivančič Kutin and S. Ledinek (2015). Le collezioni uniscono. Zbirke povezukejo. Collezioni ethologiche, tradizione orale e turismo culturale fra le Alpi e il Carso. Udine: Università degli Studi di Udine.

"Derrida, J. (1997). Mal de archivo. Una impresión freudiana. Madrid: Trotta.

»Dolenc, J. (2013). Manuscript collection of Slovenian Folklore . Ljubljana: Zrc-Sazu.

» Fernández Latour de Botas, O. (1962). Cantares históricos de la tradición argentina. Buenos Aires: Instituto Nacional de Investigaciones Folklóricas.

"García, S. y D. Rolandi. (2000). Cuentos de las tres abuelas. Buenos Aires: Unesco.

" Giovannoni, N. y M. Poduje. (1988). Cuentos y Leyendas de La Pampa. Santa Rosa: Dirección General de Cultura de La Pampa.

» González, A. et al. (2018). Atlas lingüístico y etnográfico del Nuevo Cuyo I y II. Buenos Aires: Instituto Geográfico Nacional.

» González, A., E. Mercado Lobos y A. Quinteros de Silva. (2010). Fondo Berta Vidal de Battini FONVIBA: estudios lingüístico-etnográficos en el contexto del Bicentenario de la Revolución de Mayo. San Juan: Universidad Nacional de San Juan.

» González, A. y N. García (en prensa). “The Berta Vidal de Battini archive, heritage of the National University of San Juan, Argentina". En: Palleiro, M. (ed.), Argentinean and Slovenian Folk Narrative Archives. Buenos Aires: Instituto de Investigaciones Lingüísticas y Filológicas “Manuel Alvar”.

" Grimm, J. y Grimm W. (1857). Kinder und Hausmärchen. Göttingen. Verlag der Dieterichschen Buchhandlung.

"Hansen, T. (1957). The types of the foktale in Cuba, Puerto Rico, the Dominican Republic, and Spanish America. Berkeley \& Los Angeles: University of California Press.

"Ivančič Kutin, B. (2018). Krivopete. Ljubljana: Zrc- Sazu.

"Ivančič Kutin, B. (en prensa). "On the context and texture of folklore narratives: a survey among the collaborators of the Glasovi collection". En: Palleiro, M. (ed.), Argentinean and Slovenian Folk Narrative Archives. Buenos Aires: Instituto de Investigaciones Lingüísticas y Filológicas “Manuel Alvar”.

»Ivančič Kutin, B. (Manuscrito cedido por la autora). "Archivos de narrativa tradicional eslovena en el contexto mundial". Conferencia dictada el 4 octubre de 2019 en el Instituto de Filología y Literaturas Hispánicas “Amado Alonso", Universidad de Buenos Aires.

"Ivančič Kutin, B. (en prensa). "The students archive of the slavist, folklorist and ethnologist Janez Dolenc“. En: Palleiro, M. (ed.), Argentinean and Slovenian Folk Narrative Archives. Buenos Aires: Instituto de Investigaciones Lingüísticas y Filológicas “Manuel Alvar”. 
»Koiva, M. \& A. Kuperjanov (2004). "Folklore studies and Ethnology in Slovenia 1". Folklore, 27, 185-192.

» Kõiva, M. \& A. Kuperjanov (2005). "Folklore studies and ethnology in Slovenia 2", Folklore, 29, 1-12. En: www.folklore.ee ' folklore ' vol29 (2005), obtenido el 05/03/2020.

» Kornfeld, L. (2013). “El español de la Argentina: descripción de las variedades y políticas sobre la lengua". En: I Congreso Regional de la Cátedra UNESCO en Lectura y Escritura: "Cultura Escrita y Políticas Pedagógicas en las Sociedades Latinoamericanas Actuales”. Los Polvorines: Universidad Nacional de General Sarmiento.

» Križnik, G. (1875). "O ajdovski deklici in copernicah/iz Motnikal. Odgovor na družtvena pitanja od Gašpara Križnika, črevljara”. Arkiv za povjesticu Jugoslovensku, XII(7), 142-149.

» Kropej Telban, M. (2012). Supernatural beings from Slovenian myth and folktales. Ljubljana: Zrc.

" Kropej Telban, M. (2015). Tipni indeks slovenskih ljudskih pravljic. (Type Index of Slovenian Folktales. Animal Tales and Fables) Ljubljana: Založba-Zrc.

"Kropej Telban, M. (2017). "Karel Štrekelj and his unpublished collection of Slovenian folktales". Fabula, 58, No. 3-4, 271-293.

»Kropej Telban, M. et al. (2010). A treasury of Slovenian Folklore. 101 Folk Tales from Slovenia. Rodovljica: Didakta.

» Kropej Telban, M. (en prensa). "Slovenian Folklore Archive from the 19th Century - Karel Štrekelj’s Legacy”. En: Palleiro, M. (ed.), Argentinean and Slovenian Folk Narrative Archives. Buenos Aires: Instituto de Investigaciones Lingüísticas y Filológicas “Manuel Alvar”.

"Lorbek, T. (en prensa). “Comentario de las contribuciones de las investigadoras eslovenas". En: Palleiro, M. (ed.), Argentinean and Slovenian Folk Narrative Archives. Buenos Aires: Instituto de Investigaciones Lingüísticas y Filológicas "Manuel Alvar".

»Matičetov, M. (1964). “Scritti Resiani”. Ricerche Slavistiche, 12, 123-144.

"Noia Campos, C. (2010). Catálogo tipológico do conto galego de tradición oral. Clasificación, antología y bibliografía. Vigo: Servicio de Publicaciones Universidad de Vigo.

»Palleiro, M. (1990a). "El escondite mágico" y otros cuentos folklóricos riojanos. Buenos Aires: Del Dock.

»Palleiro, M. (1990b). Estudios de Narrativa Folklórica. Buenos Aires: Filofalsía.

»Palleiro, M. (1992a). “Los tres pelos del diablo." Cuentos maravillosos de la cultura popular argentina. Buenos Aires: Del Sol.

» Palleiro, M. (1992b). Nuevos Estudios de Narrativa Folklórica. Buenos Aires: Rundinuskin.

» Palleiro, M. (1998). La fiesta en el cielo. Cuentos populares de animales. Buenos Aires: Del Sol.

»Palleiro, M. (2004). Fue una historia real. Itinerarios de un archivo. Buenos Aires: Instituto de Filología y Literaturas Hispánicas “Amado Alonso", Universidad de Buenos Aires.

»Palleiro, M. (2008). “Archives of Argentinean Folk Narrative: trends, topics and history of Argentinean Folkloristics". International Society for Folk Narrative Research Newsletter, 6, 20-28. 
»Palleiro, M. (2011). Jornada "Archivos de Narrativa Tradicional Argentina" ANATRA. Buenos Aires: Instituto de Filología y Literaturas Hispánicas “Amado Alonso", Universidad de Buenos Aires.

» Palleiro, M. (2011). "La fuga mágica. Cuentos maravillosos de la provincia de La Rioja, Argentina". En: Palleiro, M. (ed.), Jornada "Archivos de Narrativa Tradicional Argentina" (ANATRA). Buenos Aires: Instituto de Filología y Literaturas Hispánicas “Amado Alonso", Universidad de Buenos Aires, CD adjunto.

"Palleiro, M. (2013). "Cuento folklórico y narrativa oral: versiones, variantes y estudios de génesis". Cuadernos Lirico, 9. En: http://lirico.revues.org/1120; obtenido el 10/02/2020.

»Palleiro, M. (2014). "Archivos de Narrativa Tradicional Argentina 1921-2005”. En: Palleiro, M. (ed.), Oralidad, narrativa y archivos: tradición y cambio social en el contexto argentino. Buenos Aires: Facultad de Filosofía y Letras, Universidad de Buenos Aires, 67-104.

»Palleiro, M. y V. Civila Orellana (2015). “Folklore argentino, italiano y esloveno: notas para una aproximación comparativa al estudio de identidades migrantes". En: Giusto, V. (ed.), Folklore Latinoamericano XV. Buenos Aires: Universidad Nacional de las Artes, 571-600.

»Palleiro, M. (2015). “Vidal de Battini, Berta Elena”. En: Enzyklopaedie des Maerchens 14. Berlin: De Gruyter, 1843-1845.

»Palleiro, M. (2016). El cuento folklórico riojano: una aproximación a la narrativa oral. Buenos Aires: La Bicicleta.

"Palleiro, M. (2017). "My visit to the Institute of Ethnology of the Slovenian Academy of Sciences and Arts in Ljubljana". Slovstvena Folkloristika, 14-15, 39-42.

»Palleiro, M. (2018). La dama fantasma. Los laberintos de la memoria en el relato folklórico. Buenos Aires: La Bicicleta.

»Palleiro, M. (2019a). “Animals, tale types and belief narratives in Argentinean folklore". Folklore, 77, 11-38.

»Palleiro, M. (ed). (en prensa). Argentinean and Slovenian Folk Narrative Archives. Buenos Aires: Instituto de Investigaciones Lingüísticas y Filológicas "Manuel Alvar".

»Palleiro M. y M. Peltzer (en prensa). "The lady ghost in Argentinean urban graveyards: Dark tourism and ghostly narrative itineraries". En: Merriman, K. (ed.), The thrill of the Dark: Heritages of Fear, Fascination and Fantasy. Birmingham: University of Birmingham Press.

» Šavli, J. (2004). Slovenia: Discovering a European Nation. Udine: Humar.

»Skubik, M. (2015). “Commento folklorico di Jan Baudouin de Courtenay, Materiali per la dialettologia e l'etnografia slava meridionale a cura di Liliana Spinozzi Monai”, Linguistica, 28, No.1, 167-168Stanonik, M. (2009). Le Folklore littéraire. Approche pluridisciplinaire d'un phénomène syncrétique. Paris: L' Harmattan.

»Stanonik, M. (ed.) (1988-2020). Glasovi (Voices). Ljubljana: Zrc-Sazu.

»Stanonik, M. Et al. (2008). Gašper Križnik (1848-1904) in njegov čas. Ljubljana : Institut za slovensko narodopisje ZRC SAZU.

» Štrekelj, K. (1895-1923). Slovenske narodne pesmi (Slovenian Folk Songs) I-IV. Ljubljana: Slovenska matica. 
" Thompson, S. (1946). The folktale. New York: Dryden Press.

» Thompson, S. (1955-1958). Motif-Index of Folk-Literature. A Classification of Narrative Elements in Folk-Tales, Ballads, Myths, Fables, Mediœeval Romances, Exempla, Fabliaux, Jest-Books and Local Legends. Bloomington: Indiana University Press.

»Uther, H. (2011 [2004]). The types of International Folktales: a classification and bibliography, based on the system of Antti Aarne and Stith Thompson. Helsinki: Academia Scientiarum Fennica.

"Vidal de Battini, B.E. (1980-1984). Cuentos y leyendas populares de la Argentina I-IX. Buenos Aires: Ediciones Culturales Argentinas.

"Vidal de Battini, B.E. (1995). Cuentos y leyendas populares de la Argentina X. Buenos Aires: Secretaría de Cultura, Ministerio de Educación y Justicia de la Nación.

»Vidal de Battini, B.E. (1949). El Habla Rural de San Luis. Buenos Aires: Facultad de Filosofía y Letras, Universidad de Buenos Aires.

»Vidal de Battini, B.E. (1964). El español de la Argentina. Buenos Aires: Consejo Nacional de Educación. 